INPLASY

PROTOCOL

To cite: Li et al. Comparison of multiple acupoints combination in the treatment of Post-stroke Cognitive Impairment: a network metaanalysis. Inplasy protocol 202130054. doi: 10.37766/inplasy2021.3.0054

Received: 15 March 2021

Published: 16 March 2021

Corresponding author: Qian Yu

yqswx11@163.com

Author Affiliation: The Hospital of Chengdu University of Traditional Chinese Medicine

Support: No fund.

Review Stage at time of this submission: Data analysis.

Conflicts of interest:

None declared.

\section{Comparison of multiple acupoints combination in the treatment of Post-stroke Cognitive Impairment: a network meta-analysis}

\author{
Li, RY1; Huang, RJ2; Chen, KY33 Yu, Q44.
}

Review question / Objective: Acupuncture is one of the traditional Chinese medical methods in my country, and its curative effect has been widely recognized at home and abroad. There have been many clinical studies that have confirmed that acupuncture can improve cognitive dysfunction after stroke. The theoretical system and compatibility of acupuncture points are diverse, and the effects are also uneven. In this study, the Network Metaanalysis method was used for the Mini-mental State Examination (MMSE), Montreal Cognitive Assessment (MoCA), ADL-Barthel Index (Barthel in dex, BI) Score and the total effective rate of clinical treatment are 4 indicators to evaluate the effect of various acupuncture treatments on PSCI.

INPLASY registration number: This protocol was registered with the International Platform of Registered Systematic Review and Meta-Analysis Protocols (INPLASY) on 16 March 2021 and was last updated on 16 March 2021 (registration number INPLASY202130054).

\section{INTRODUCTION}

Review question / Objective: Acupuncture is one of the traditional Chinese medical methods in my country, and its curative effect has been widely recognized at home and abroad. There have been many clinical studies that have confirmed that acupuncture can improve cognitive dysfunction after stroke. The theoretical system and compatibility of acupuncture points are diverse, and the effects are also uneven. In this study, the Network Metaanalysis method was used for the Mini- 
mental State Examination (MMSE), Montreal Cognitive Assessment (MoCA), ADL-Barthel Index (Barthel in dex, BI) Score and the total effective rate of clinical treatment are 4 indicators to evaluate the effect of various acupuncture treatments on PSCI.

Condition being studied: Stroke, is a common acute cerebrovascular disease in middle-aged and elderly people. The mortality rate accounts for about $30 \%$ of the total incidence. The cause is mostly abnormal cerebral microcirculation caused by sudden rupture or blockage of cerebral blood vessels. , the huge social burden, high mortality and high morbidity make its health costs rise exponentially. As we all know, the development of stroke to a serious stage is not only accompanied by language and movement disorders, but moreover, it will lead to a reduction in daily life self-care ability and a reduction in the scope of daily interaction, which will cause a series of knock-on effects such as reduced contact with the outside world, resulting in the amount of information. Decline and decreased brain activity function will lead to the decline of brain cognitive function, that is, post-stroke cognitive impairment (PSCI). With the improvement and optimization of the world medical level, the mortality of stroke patients The mortality rate of patients is gradually decreasing, and cognitive impairment after stroke is still the main problem that affects the life and work of patients after recovery, and the main cause of social and family.

\section{METHODS}

Participant or population: Adults with PSCI will be included.

Intervention: Acupuncture therapy with special points was the main intervention(Zishen Yisui acupuncture therapy,Tiaoshen Tongluo acupuncture therapy).

Comparator: Acupuncture therapy with common points was the main intervention.
Study designs to be included: Randomized controlled trials (RCTs) will be included.

Eligibility criteria: Trials were excluded if they meet any of the following criteria: (1) the research subject of the literature is non-clinical patients, (2) repeated publications and (3) literature with unclear diagnostic criteria or unclear related outcome indicators.

Information sources: Two reviewers conducted a comprehensive and independent review of these publications and cross-checked them. In case of disagreement, a third party will arbitrate and evaluate. Data items extracted: title, name of the first author, baseline characteristics of the included study (sample size, patient age, treatment measures, etc.), publication year, riskrelated factors of bias (randomization method, blinding, etc.), outcome data (MMSE, MoCA, BI, Total Effectiveness). If the above data is unclear, they can try to supplement the information by contacting the corresponding author of the original text.

Main outcome(s): MMSE (Mini-mental State Examination), MoCA(Montreal Cognitive Assessment).

Additional outcome(s): The overall clinical effectiveness, BI(Barthel index).

Quality assessment / Risk of bias analysis: According to the Risk of Bias Table recommended in Cochrane Handbook 5.1.0, the quality of the included literature was evaluated, including reporting bias, detection bias, performance bias, and selection bias. bias), loss to follow-up bias (attrition bias) and other bias (other bias) 6 parts. The content of each part of the evaluation can be judged as high, medium, and low risk based on the standards in Cochrane Handbook 5.1.0. 2 results.

Strategy of data synthesis: The statistical method of the network meta-analysis is based on the frequency framework, and all the outcome indicators used the random 
effects model for data analysis. If the evaluation indicators of this study were continuous variables MMSE and MoCA, the mean difference (MD) was used as the effect size. When it is a continuous variable outcome indicator $\mathrm{BI}$, then considering that some of the literature measurement methods were improved, and the standardized mean difference (SMD) was used. If it is a binary variable, use the odds ratio (OR) as the effect size, and calculate the corresponding $95 \%$ credibility interval (Cl).

Subgroup analysis: No subgroup.

Sensitivity analysis: Use STATA 14.0 software (Stata Corporation, Lakeway, Texas, USA) to draw network evidence relationship diagrams, forest diagrams, grade probability diagrams, funnel diagrams and corresponding statistics. When testing global consistency, if the difference is not statistically significant, that is, $P>0.05$, it indicates that there is no overall inconsistency. In order to evaluate local inconsistencies, this study calculates the inconsistency factors (IF) and $95 \% \mathrm{Cl}$ of each closed loop in the network. The calculation method uses the ifplot command in Stata to perform loop inconsistency detection (loop inconsistency). The lower limit of the 95\% confidence interval contains or is close to 0 , indicating that the local comparison, that is, the direct comparison evidence, is very consistent with the indirect comparison evidence.

Language: Chinese.

Country(ies) involved: China.

Keywords: acupuncture ; post-stroke cognitive impairment; RCTs; network meta-analysis.

Contributions of each author:

Author 1 - Ruo-Yang Li.

Email: alis7718@outlook.com

Author 2 - Rui-Jue Huang.

Author 3 - Ke-Yu Chen.

Author 4 - Qian Yu.

Email: yqswx11@163.com 